\title{
Dynamical and transport properties in a family of intermittent area-preserving maps
}

\author{
Roberto Artuso, ${ }^{*}$ Lucia Cavallasca, ${ }^{\dagger}$ and Giampaolo Cristadoro \\ Center for Nonlinear and Complex Systems and Dipartimento di Fisica e Matematica, Università degli Studi dell'Insubria, \\ Via Valleggio 11, 22100 Como, Italy and Sezione di Milano, INFN, Via Celoria 16, 20133 Milano, Italy
}

(Received 19 December 2007; published 11 April 2008)

\begin{abstract}
We introduce a family of area-preserving maps representing a (nontrivial) two-dimensional extension of the Pomeau-Manneville family in one dimension. We analyze the long-time behavior of recurrence time distributions and correlations, providing analytical and numerical estimates. We study the transport properties of a suitable lift and use a probabilistic argument to derive the full spectrum of transport moments. Finally, the dynamical effects of a stochastic perturbation are considered.
\end{abstract}

DOI: 10.1103/PhysRevE.77.046206

PACS number(s): 05.45.-a

\section{INTRODUCTION}

Hamiltonian systems are generically not fully hyperbolic [1]: for example, the phase space of typical area-preserving maps reveals the coexistence of chaotic trajectories and islands of regular motion (periodic or quasiperiodic trajectories) [2]. Even when we are concerned with statistical properties of motion on the chaotic component, we cannot neglect the presence of regular structures. They deeply influence chaotic motion as, whenever trajectories come close to integrable islands, they stick there for some time, and irregular dynamics is thus punctuated by laminar segments where the system mimics an integrable one. This intermittent behavior has strong influence on the long-time properties of quantities like correlation decay or recurrence time statistics, which typically present a power-law tail [3-7]. In unbounded systems, intermittency influences transport properties, generating anomalous diffusion processes (see [8] and references therein), in contrast to the normal diffusion observed for fully hyperbolic systems [9].

While much effort is still devoted to fully understanding the general picture of mixed phase space, the situation simplifies if we let the islands of regular motion shrink to zero: even the presence of a single marginally stable fixed point can produce intermittentlike behavior [10-12]. In one dimension this corresponds to the Pomeau-Manneville maps on the unit interval [13],

$$
x_{n+1}=x_{n}+\left.x_{n}^{z}\right|_{\bmod 1}, \quad z>1,
$$

which represent one of the few examples of non-fullyhyperbolic systems for which analytic results can be obtained with a variety of different techniques (see, for instance, [14-21]). Such maps present a polynomial decay of correlations and recurrence time statistics with exponents that depend on the intermittency parameter $z[15,16,22]$. Moreover, a proper lift on the real line can generate anomalous diffusion and the set of transport moments typically shows a two-scale structure [23-26].

\footnotetext{
*roberto.artuso@uninsubria.it

†lucia.cavallasca@uninsubria.it

¥giampaolo.cristadoro@uninsubria.it
}

The situation is much less satisfactory in more than one dimension. Rigorous results were derived for specific cases, where it was possible to give precise bounds on the rate of mixing [12,27]. Here we study a family of area-preserving maps with a neutral fixed point. This family depends on a parameter that governs stability properties of the fixed point, in analogy with the Pomeau-Manneville maps.

We introduce the two-dimensional family of areapreserving maps in Sec. II, where we also discuss the dynamical features we will look at. In Sec. III we will consider the unstable manifold of the neutral fixed point, and provide simple estimates that will be pivotal in predicting the decay of survival probabilities. Section IV contains extensive investigations on survival probability and correlation functions decay. By lifting the map on an unbounded phase space we then analyze transport properties in Sec. V. The role of a stochastic perturbation is then studied in Sec. VI, while we present our conclusions in Sec. VII.

\section{THE MODEL}

We define the following one-parameter family of maps $T_{\gamma}(x, y): \mathbb{T}^{2} \rightarrow \mathbb{T}^{2}$, where $\mathbb{T}^{2}=[-\pi, \pi)^{2}$ (with torus topology):

$$
T_{\gamma}(x, y)= \begin{cases}x+f_{\gamma}(x)+y & \text { on } \mathrm{T}, \\ y+f_{\gamma}(x) & \text { on } \mathrm{T},\end{cases}
$$

with

$$
f_{\gamma}(x)=\pi \operatorname{sgn}(x)\left|\frac{x}{\pi}\right|^{\gamma}, \quad \gamma>1 .
$$

The map $T_{\gamma}$ is area preserving for every choice of the impulsive force $f(x)$. The Jacobian matrix is

$$
\mathbf{J}_{\gamma}(x, y)=\left(\begin{array}{cc}
1+f_{\gamma}^{\prime}(x) & 1 \\
f_{\gamma}^{\prime}(x) & 1
\end{array}\right)
$$

so we have $\operatorname{det} \mathbf{J}_{\gamma}(x, y)=1$ and $\operatorname{Tr} \mathbf{J}_{\gamma}(x, y)=2+f_{\gamma}^{\prime}(x)>2$ for $x \in[-\pi, \pi) /\{0\}:$ the map is everywhere hyperbolic except on the line $x=0$ and thus the fixed point at the origin is marginally stable (parabolic) for every $\gamma$. The parameter $\gamma$ changes the "stickiness" of the origin, in analogy with the intermittency exponent $z$ appearing in the Pomeau-Manneville maps of Eq. (1). We point out that the choice $f(x)=x-\sin (x)$ 
$[10,12]$ gives rise to a marginal fixed point with the same stability properties as those for $\gamma=3$ in Eq. (2). We note explicitly that for our model $f \in C^{k}$ with $k=[\gamma-1]$ (where [.] denotes the integer part) unless $\gamma$ is an odd integer, in which case $f \in C^{\infty}[28]$.

\section{A. Dynamical indicators}

In order to get information about the dynamical properties of the systems it is often useful to employ time statistics [29]. We choose a set $\Omega$ including the parabolic fixed point and then define a partition of $\Omega$ into disjoint sets $\Omega_{n}$, each representing the set of points that leave $\Omega$ in exactly $n$ iterations. The survival probability $p_{\Omega}(n)$ is the fraction of initial conditions in $\Omega$ that are still in $\Omega$ after $n$ iterations. The behavior of $p_{\Omega}(n)$ generally depends on the choice of the measure $\mu_{i}$ with which we distribute initial conditions over $\Omega$, which may be quite different from the invariant measure. In the present case the invariant measure is the Lebesgue one $\mu$, which also represents the most natural way to spread initial conditions over $\Omega$, so $\mu_{i}=\mu$ and

$$
p_{\Omega}(n)=\frac{1}{\mu(\Omega)} \sum_{k>n} \mu\left(\Omega_{n}\right) .
$$

We may also define the waiting time distribution (or residence time statistics) over $\Omega, \psi_{\Omega}(n)$, as the probability that once a trajectory enters the set $\Omega$ it stays there for exactly $n$ time steps. $\psi_{\Omega}(n)$ is computed by running a long trajectory and recording residence times in $\Omega: \psi_{\Omega}(n)$ is just the probability distribution of such residence times. In our case

$$
\psi_{\Omega}(n)=\frac{1}{\mu(\Omega)}\left[\mu\left(\Omega_{n}\right)-\mu\left(\Omega_{n+1}\right)\right]
$$

where ergodicity and the property that the map preserves the Lebesgue measure have been used. We point out that, in general, while $p_{\Omega}(n)$ depends upon an arbitrary choice of the distribution of initial conditions, $\psi_{\Omega}(n)$ does not.

From Eqs. (5) and (6) we see that the asymptotics of these quantities are tightly related [29]: in particular, if the measure of the sets $\Omega_{n}$ decays according to a power law

$$
\mu\left(\Omega_{n}\right) \sim n^{-\alpha-1},
$$

we get

$$
p_{\Omega}(n) \sim n^{-\alpha}
$$

and

$$
\psi_{\Omega}(n) \sim n^{-\alpha-2} .
$$

Besides their intrinsic interest, these relations have remarkable links with the problem of establishing the mixing rates for the system $[4,5,30,31]$. We briefly recall it with a simple argument: suppose we consider an observable $A$ that remains fully correlated for portions of trajectories within $\Omega$, and otherwise completely decorrelated (due to randomness of motion outside of $\Omega$ ); then we may easily show that [31]

$$
C_{A A}(n)=\langle A(n) A(0)\rangle-\langle A\rangle^{2} \sim\left(\left\langle A^{2}\right\rangle-\langle A\rangle^{2}\right) \sum_{m=n}^{\infty} \sum_{k=m}^{\infty} \psi_{\Omega}(k) ;
$$

so that the exponents of the power-law decay of survival probability and correlations should coincide,

$$
C_{A A}(n) \sim n^{-\alpha} .
$$

We observe that the validity of Eq. (10) has been carefully scrutinized numerically for chaotic billiards [31,32], and even validated in rigorous estimates of polynomial mixing speed for one-dimensional (1D) intermittent systems [15] (see also [33]), but indications of its possible failure have also been suggested [34].

It is interesting to remark that showing that the power-law decays of $p_{\Omega}(n)$ and $\psi_{\Omega}(n)$ differ by 2 [Eqs. (8) and (9)] employs the fact that the Lebesgue measure is the invariant one for the system. Actually, for the map of Eq. (1), where the invariant measure is not uniform, they differ by 1 if we choose the initial conditions uniformly distributed with Lebesgue measure (while the exponent of the waiting time distribution coincides with the one ruling the length of the segments $\Omega_{n}$ [13]). A relationship coinciding with Eqs. (8) and (9) holds instead for another intermittent map, introduced by Pikovsky [24] (some features of this map are also described in [35]):

$$
x_{n+1}=\tilde{f}_{z}\left(x_{n}\right),
$$

where $\tilde{f}_{z}$ is an (odd) circle map, again dependent on an intermittency parameter $z$, implicitly defined on the torus $T$ $=[-1,1)$ by

$$
x= \begin{cases}\frac{1}{2 z}\left[1+\tilde{f}_{z}(x)\right]^{z}, & 0<x<1 /(2 z), \\ \tilde{f}_{z}(x)+\frac{1}{2 z}\left[1-\tilde{f}_{z}(x)\right]^{z}, & 1 /(2 z)<x<1 .\end{cases}
$$

A key feature that the map of Eq. (12) shares with our model is that the invariant distribution is smooth, coinciding with the Lebesgue measure, as can be checked by inspection of the form of the Perron-Frobenius operator.

For the map under consideration it is possible to get an estimate of the exponent $\alpha$ by studying the invariant manifolds of the parabolic fixed point.

\section{INVARIANT MANIFOLDS}

A typical trajectory staying for a long time in $\Omega$ (again we take as $\Omega$ a region including the parabolic fixed point) enters $\Omega$ close to the stable manifold, escaping along the unstable one (Fig. 1). In particular, we are going to discuss how trajectories escape by following the unstable manifold of the marginal fixed point. For the odd symmetry of $f_{\gamma}(x)$, we can restrict the analysis to the first quadrant.

Let us call $(x, y(x))$ the graph of the unstable manifold in the neighborhood of the origin and suppose that, very close to the marginally stable fixed point, $y(x) \simeq x^{\sigma}$. With the 


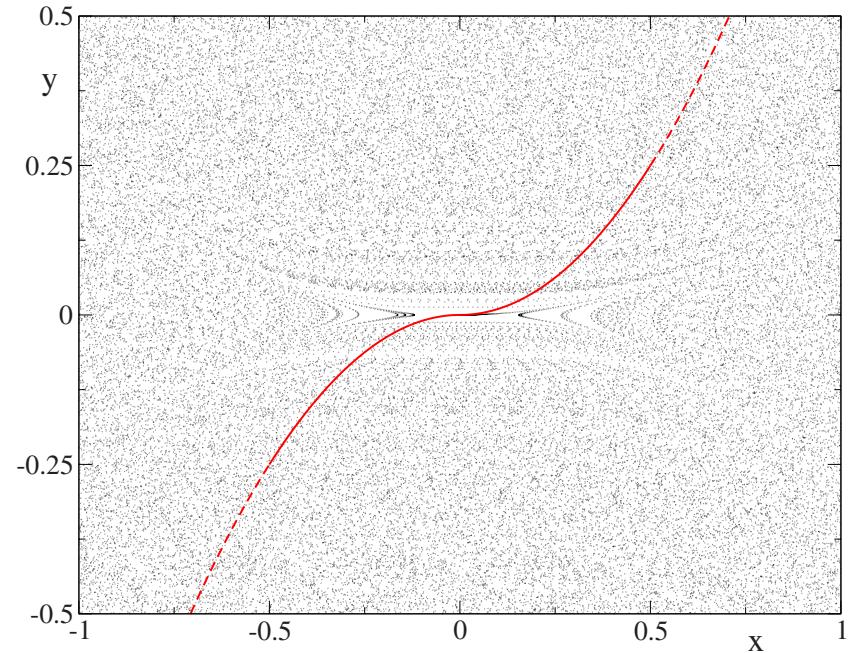

FIG. 1. (Color online) Portion of phase space of the map of Eq. (2) for $\gamma=3$ close to the marginal fixed point, together with the graph of its unstable manifold [continuous (red) line].

choice of Eq. (3) we have that $f_{\gamma}(x) \simeq a x^{\gamma}$ and the Jacobian matrix [Eq. (4)] of the map is

$$
\mathbf{J}_{\gamma}(x, y)=\left(\begin{array}{cc}
1+b x^{\gamma-1} & 1 \\
b x^{\gamma-1} & 1
\end{array}\right),
$$

whose eigenvalues are written to leading order as $\lambda_{ \pm}$ $=1 \pm \beta x^{(\gamma-1) / 2}$.

The vector $\left(1, y^{\prime}(x)\right)$, i.e., $\left(1, \eta x^{\sigma-1}\right)$, tangent to the unstable manifold satisfies

$$
\mathbf{J}_{\gamma}(x, y)\left(\begin{array}{c}
1 \\
\eta x^{\sigma-1}
\end{array}\right) \simeq\left(1+\beta x^{(\gamma-1) / 2}\right)\left(\begin{array}{c}
1 \\
\eta x^{\sigma-1}
\end{array}\right)
$$

i.e.,

$$
\begin{gathered}
1+b x^{\gamma-1}+\eta x^{\sigma-1} \simeq 1+\beta x^{(\gamma-1) / 2}, \\
b \quad x^{\gamma-1}+\eta x^{\sigma-1} \simeq \eta x^{\sigma-1}+\eta \beta x^{(\gamma-1) / 2+(\sigma-1)}
\end{gathered}
$$

and from these equations, remembering that $x \ll 1$ and $\gamma$ $>1$, we obtain

$$
\sigma=\frac{\gamma+1}{2} .
$$

We note explicitly that, for $\gamma=3$, this result is in agreement with the case $f=x-\sin (x)$ derived in [12]. We can now study the dynamics restricted to the unstable manifold. Let us denote by $\ell$ the arc length coordinate along the manifold; for small $x$ we get

$$
\ell(x)=\int^{x} d x \sqrt{1+[d y(x) / d x]^{2}} \simeq x .
$$

Denote by $\ell_{n}$ the coordinate $\ell$ at a point $\left(x_{n}, y\left(x_{n}\right)\right)$ and by $\ell_{n+1}$ the coordinate along the manifold of $T_{\gamma}\left(x_{n}, y\left(x_{n}\right)\right)$,

$$
\ell_{n+1}=\ell_{n}+h\left(\ell_{n}\right) .
$$

By using Eqs. (18) and (2) we get

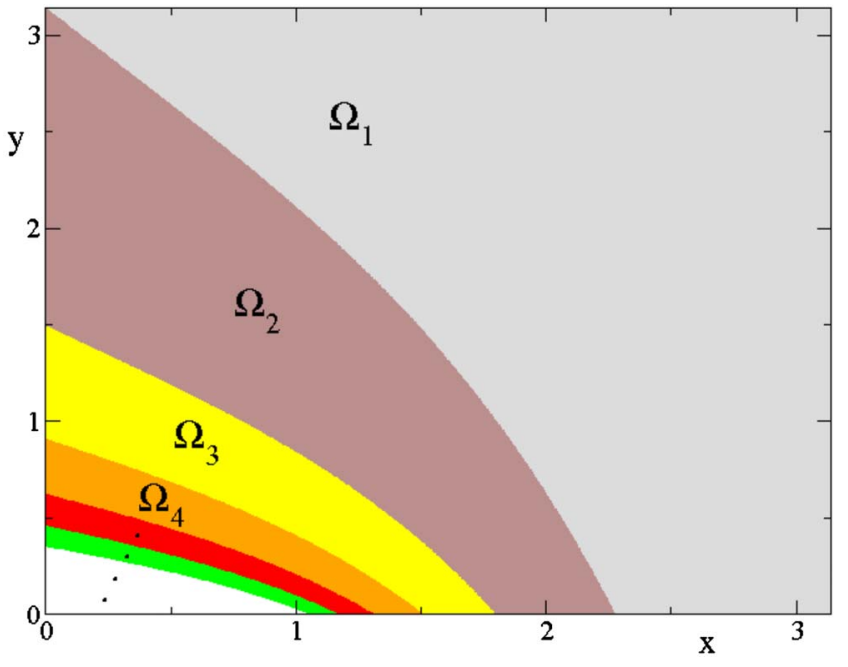

FIG. 2. (Color online) A few $\Omega_{n}$ (once we set $\Omega$ as the first quadrant $x \geq 0, y \geq 0$ ).

$$
h(\ell) \simeq \frac{d \ell}{d t}(\ell)=\frac{d \ell}{d x} \frac{d x}{d t}(\ell) \simeq\left[y(\ell)+x^{\gamma}(\ell)\right]=\ell^{\sigma}+\ell^{\gamma} .
$$

From Eq. (17) and by remembering that $\gamma>1$, we obtain, via a continuous time approximation [36,37],

$$
h(\ell) \simeq \ell^{\sigma}=\frac{d \ell}{d t} .
$$

If we fix the boundary of $\Omega$ at a scale $L$, we can then evaluate the time needed to reach the boundary as a function of the arc length $\ell$ along the manifold, by employing the standard argument of $[36,37]$ :

$$
T(\ell)=\frac{2}{\gamma-1}\left(\ell^{-(\gamma-1) / 2}-L^{-(\gamma-1) / 2}\right) ;
$$

this implies the following scaling for the inverse function:

$$
\ell(T) \sim T^{-2 /(\gamma-1)} .
$$

We now arrive at the crucial point: we estimate $p_{\Omega}(n)$ as the area of a rectangle having one vertex at the origin (the parabolic fixed point), and another at a point on the unstable manifold $(\bar{x}, \bar{y})$ that exits $\Omega$ in $n$ steps (see Fig. 2):

$$
p_{\Omega}(n) \simeq \overline{x y} \simeq \ell(n)^{\sigma+1} \simeq\left(n^{-2 /(\gamma-1)}\right)^{\sigma+1}=n^{-(\gamma+3) /(\gamma-1)} .
$$

Thus we have an estimate of the power-law decay of the survival probability as a function of the intermittency parameter $\gamma$ as

$$
p_{\Omega}(n) \sim n^{-(\gamma+3) /(\gamma-1)},
$$

and for the waiting time distribution as well [Eq. (9)]:

$$
\psi_{\Omega}(n) \sim n^{-(3 \gamma+1) /(\gamma-1)} .
$$

In view of the argument we earlier mentioned [see Eq. (11)], the estimate of Eq. (25) suggests the same decay law for (auto)correlation functions, 


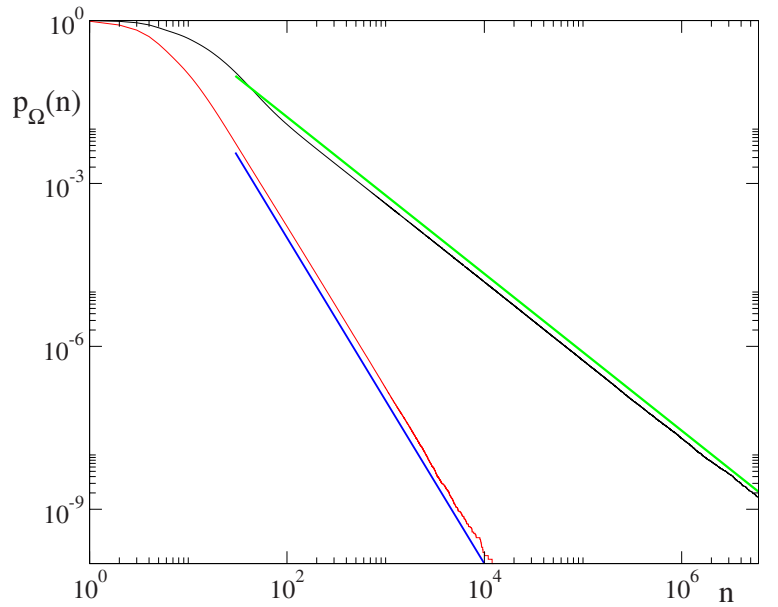

FIG. 3. (Color online) Survival probabilities for $\gamma=3$ (lower curve) and 10 (upper curve) together with the power-law decays predicted by Eq. (25). We used $10^{12}$ initial conditions and set $\Omega$ $=[-1 / 2,1 / 2]^{2}$.

$$
C_{A A}(n) \sim n^{-(\gamma+3) /(\gamma-1)} .
$$

The next section will present several numerical simulations concerning these quantities.

\section{ASYMPTOTIC DECAYS}

We start by considering the survival probability: Fig. 3 shows two examples of numerically computed $p_{\Omega}(n)$. The numerical data exhibit an excellent agreement with analytic estimates over a wide range of intermittency parameters, as shown in Fig. 4, which also provides clear indications of the validity of our estimate for the asymptotic decay of the waiting time distribution.

We already mentioned that various arguments support the expectation that correlation functions should decay as the

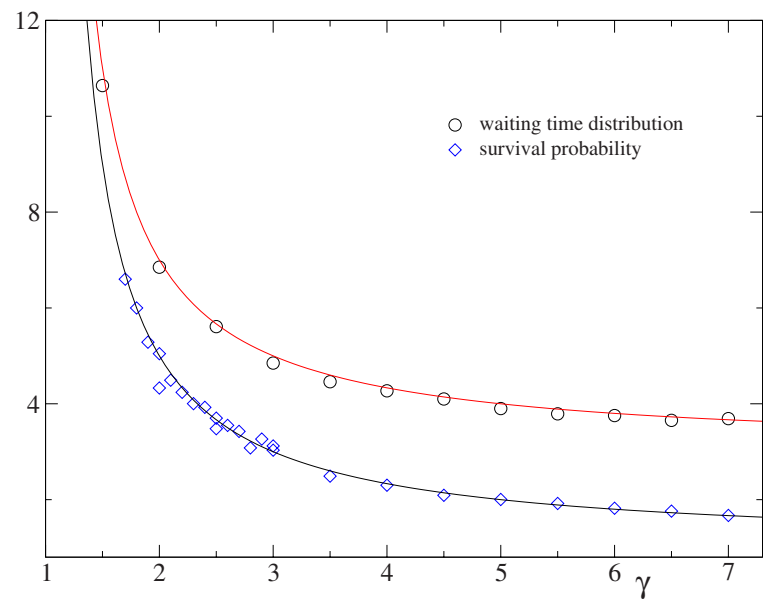

FIG. 4. (Color online) Exponents of power-law decay for survival probability $p_{\Omega}(n)$ and waiting time distribution $\psi_{\Omega}(n)$ : lines refer to analytic estimates of Eq. (25) (upper) and Eq. (26) (lower): circles come from numerical simulations of the waiting time distribution, diamonds from survival probability simulations.

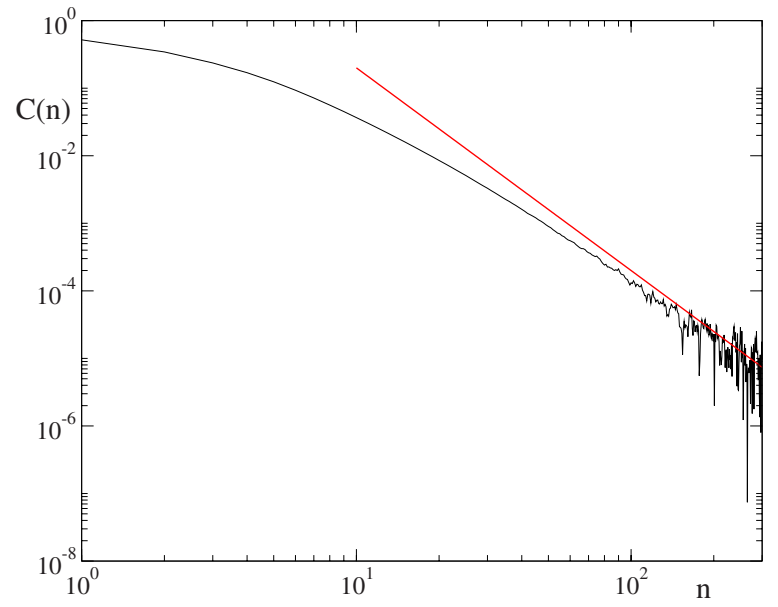

FIG. 5. (Color online) Autocorrelation function for the observable $e^{-y^{2}}$ and $\gamma=3$. We used $2 \times 10^{10}$ initial conditions (uniformly distributed in the torus cell). The predicted decay is shown by the (red) straight line, which has a slope -3 .

survival probability [Eq. (27)], so we proceed to scrutinize this prediction by running extensive direct numerical simulations on autocorrelation functions; as we are dealing with an ergodic (and mixing [12]) system, autocorrelation functions can be evaluated in terms of phase space averages (instead of temporal averages):

$$
C_{A A}(n)=\int_{\mathcal{M}} d \mu(z) A\left(T_{\gamma}^{n} z\right) A(z)-\left(\int_{\mathcal{M}} d \mu(z) A(z)\right)^{2},
$$

where $A$ is a smooth function on the phase space $\mathcal{M}$ and $\mu$ is the invariant Lebesgue measure. From a numerical point of view, it is known that often Monte Carlo evaluation of Eq. (28) cannot be pushed too far, as the statistical error is of order $1 / \sqrt{N}$ in the number of initial conditions. Generally we also expect an (exponential) transient in the decay $[10,31,38]$; the transient time $\bar{t}$ might depend on both $\gamma$ and the choice of phase space function $A$ [6]. We also remark that the smoothness of the function $A$ plays a fundamental role; as a matter of fact, we may obtain arbitrarily slow correlation decay even for Anosov maps by using integrable nonsmooth functions [39], or, from a mathematical point of view, we may have that the degree of smoothness determines the essential spectral radius of the Perron-Frobenius operator [40].

We performed explicit calculations of the autocorrelation function for different values of the intermittency parameter $\gamma$ and for different observables. We obtained the best results (i.e., cleanest curves and shortest time $\vec{t}$ ) for large values of $\gamma$ and by using $A(x, y)=e^{-y^{2}}$. The choice of the smooth function to use is quite arbitrary; we looked for a function not vanishing in correspondence with the marginal fixed point (as suggested, for example, in [6]) and by the special choice of a Gaussian depending on a single variable we could save computational time (see also [21]). In Figs. 5 and 6 we present results for $\gamma=3$ and 10 . 


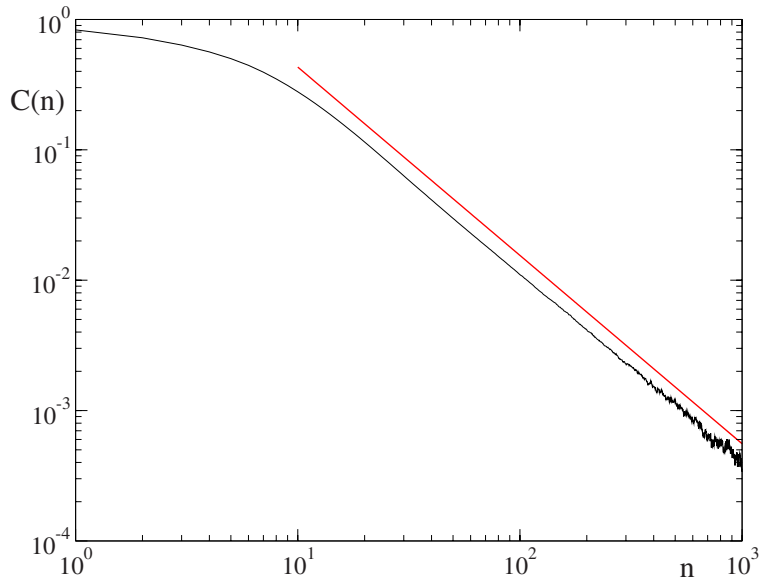

FIG. 6. (Color online) Autocorrelation function for the observable $e^{-y^{2}}$ and $\gamma=10$. We used $10^{9}$ initial conditions (uniformly distributed in the torus cell). The predicted decay is shown by the (red) straight line, which has a slope $-13 / 9$.

The case reported in Fig. 5 is important because for a similar 2D mapping (having the same intermittent exponent $\gamma=3$ ) it was proved in [12] that the decay is faster than $n^{-2}$, and a class of cross correlation was constructed indicating that the bound is optimal; while survival probability data for the same exponent indicate clearly that the decay we predict $\left(n^{-3}\right)$ is numerically well reproduced, data for correlations are less conclusive (see Fig. 5). In general, numerical data are more difficult to interpret for low values of $\gamma$, and numerical fits tend to lie below the predicted exponents (see Fig. 7), while for larger values of $\gamma$ the accordance with our estimates is much better. Moreover, the agreement improves when the number of initial conditions is increased, so that we expect the discrepancy to be essentially due to numerical limitations (Fig. 7). Further indications of the similarity be-

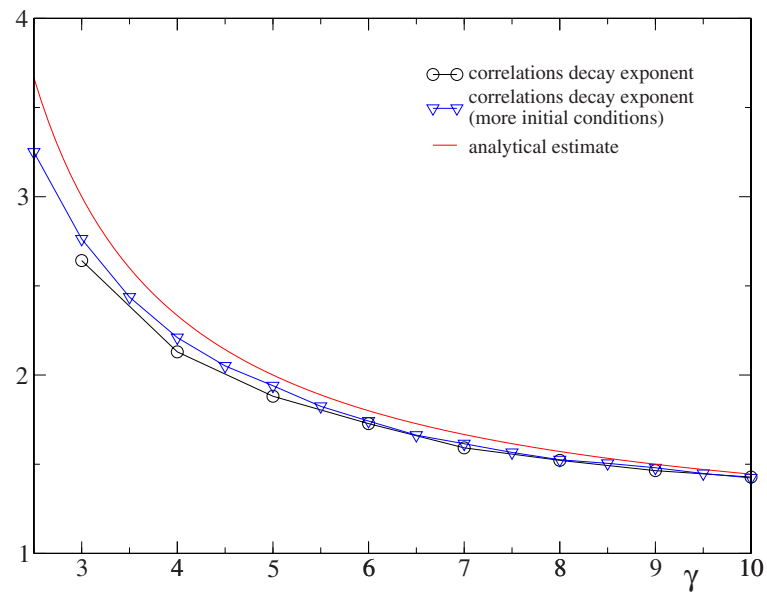

FIG. 7. (Color online) Numerical values of power-law decay exponents for the autocorrelation function for the observable $e^{-y^{2}}$ [(black) circles and (blue) triangles] together with the analytical estimates [full (red) line]. Circles were obtained by using $2 \times 10^{9}$ initial conditions while the triangles were obtained for $\gamma=2.5$ by using $5 \times 10^{10}$ initial conditions, in the range $3 \leq \gamma \leq 6$ by using 2 $\times 10^{10}$ initial conditions, and in the range $6.5 \leq \gamma \leq 10$ by using $10^{10}$ initial conditions.
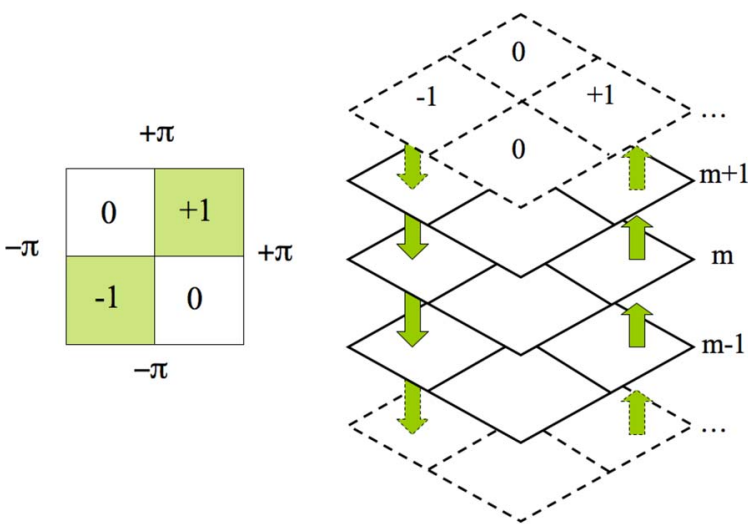

FIG. 8. (Color online) Jumping numbers and lifted map.

tween correlations and survival probability decays will be provided in Sec. VI, when we consider the role of stochastic perturbations.

\section{TRANSPORT PROPERTIES}

In order to study transport properties, we have to abandon the dynamics restricted to the torus [Eq. (2)] by lifting the map in an appropriate way.

For the sake of clarity we introduce a third dimension (say $z$ ) to describe the motion through elementary cells. We then assign a jumping number +1 to the points belonging to the first quadrant, -1 to the points belonging to the third quadrant, and 0 to all the other points. This means that a laminar phase of length $n$ will correspond to a jump in the positive direction of $n$ elementary cells Fig. 8 .

Formally, the lift is given by the following formula:

$$
\bar{T}_{\gamma}(x, y, m)=\left\{\begin{array}{cc}
\left(T_{\gamma}(x, y), m\right) & \text { for } x y<0, \\
\left(T_{\gamma}(x, y), m+\operatorname{sgn}(x)\right) & \text { for } x y \geq 0,
\end{array}\right.
$$

where $m$ is an integer variable.

Considering successive entrances into the laminar regions as uncorrelated, we can approximate the diffusion process by a continuous time random walk (CTRW) [41,42], with the probability distribution of the laminar phases given by the waiting time distribution $\psi_{\Omega}(n)$. In particular, by making use of the CTRW approach, it is possible to characterize the transport properties of the process in terms of the set of moments of the diffusing variable [43]:

$$
\left\langle|z(n)-z(0)|^{q}\right\rangle \simeq n^{\nu(q)},
$$

which is expected to present a sort of phase transition [25].

\section{A. Continuous time random walk approach}

For completeness, we briefly recall the standard theory of continuous time random walks [41-45]. Generally speaking, a CTRW is a stochastic model in which steps of a simple random walk take place at times $t_{i}$, following some waiting time distribution. Mathematically, it is asserted that a CTRW is a (non-Markovian) process subordinated to a random walk 
under the operational time defined by the process $t_{i}$ [44].

A CTRW is completely characterized by the quantity $\psi(r, \tau)$, the probability density function for moving a distance $r$ during a time interval $\tau$ in a single motion event; the dependence upon $r$ and $\tau$ can be either decoupled [i.e., $\psi(r, \tau)=\chi(r) \wp(\tau)]$ or coupled [e.g., $\psi(r, \tau)=\chi(r \mid \tau) \wp(\tau)]$.

The object we are interested in is the probability density function $P(x, t)$ of being at $x$ at time $t$; indeed it allows us to obtain the full spectrum of transport moments, through the formula

$$
\left\langle x(t)^{q}\right\rangle=(i)^{q} L^{-1}\left(\left.\frac{\partial^{q}}{\partial k^{q}} \tilde{\hat{P}}(k, u)\right|_{k=0}\right),
$$

where $L^{-1}$ is the inverse Laplace transform and $\tilde{\hat{P}}$ denotes the Fourier-Laplace transform, $k$ being the Fourier variable and $u$ the Laplace variable.

Let us introduce $\phi(x, t)$, the probability density function of passing through $(x, t)$, even without stopping at $x$, in a single motion event,

$$
\phi(x, t)=P(x \mid t) \int_{t}^{\infty} d \tau \int_{|x|}^{\infty} d r \psi(r, \tau) .
$$

$P(x, t)$ is given by the sum of the probabilities of passing through $(x, t)$, even without stopping at $x$, in one or more motion events,

$$
P(x, t)=\phi(x, t)+\int_{-\infty}^{\infty} d x^{\prime} \int_{0}^{t} d \tau \psi\left(x^{\prime}, \tau\right) \phi\left(x-x^{\prime}, t-\tau\right)+\cdots .
$$

By performing the convolution integrals, the Fourier-Laplace transform of this expression assumes the closed form

$$
\tilde{\hat{P}}(k, u)=\frac{\tilde{\hat{\phi}}(k, u)}{1-\tilde{\hat{\psi}}(k, u)} .
$$

A special realization of the CTRW is the so-called velocity model [42]: a particle moves at a constant velocity for a given time, then stops and chooses a new direction and a new time of sojourn at random according to given probabilities.

Our case belongs to this class, with velocities being \pm 1 , and

$$
\chi(r \mid \tau)=\frac{1}{2} \delta(|r|-\tau) \quad \text { and } \quad \wp(\tau) \sim \tau^{-g},
$$

so that

$$
\psi(r, \tau) \sim \frac{1}{2} \delta(|r|-\tau) \tau^{-g} \quad \text { and } \quad \phi(x, t) \sim \frac{1}{2} \delta(|x|-t) t^{-g+1},
$$

where $g=\frac{3 \gamma+1}{\gamma-1}, \wp(\tau)$ being given by the waiting time distribution $\psi_{\Omega}(n)$ of Eq. (26).

By making use of the Tauberian theorems for the Laplace transform [46] and by applying the CTRW formalism [45] we derive, through Eqs. (31) and (34) the full spectrum of

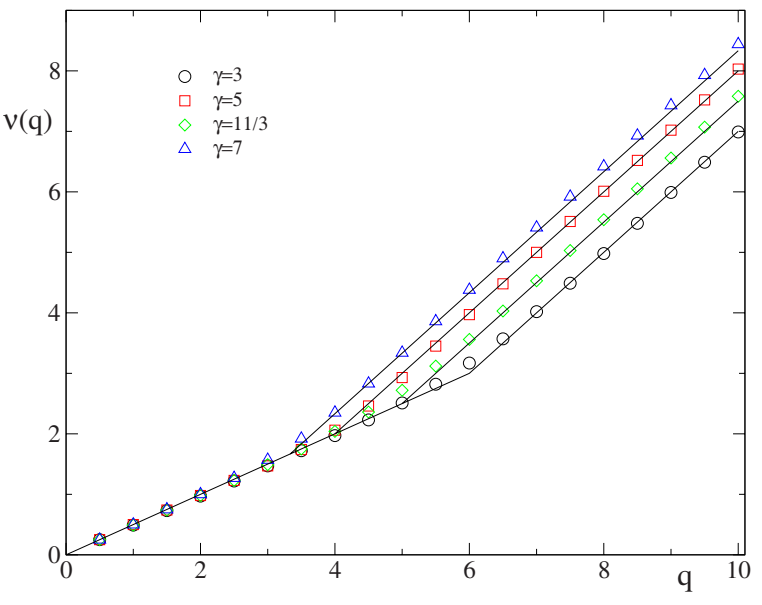

FIG. 9. (Color online) Spectrum of the transport moments for different values of the parameter $\gamma$. Lines correspond to theoretical predictions of Eq. (38); symbols correspond to numerical simulations: circles $\gamma=3$, diamonds $\gamma=11 / 3$, squares $\gamma=5$, and triangles $\gamma=7$.

transport moments. The obtained spectrum of moments (more precisely, from the previous calculation it is possible to obtain only the even moments, and then to infer that a similar law drives also the behavior of the absolute value of odd moments) is

$$
\left\langle|z(n)-z(0)|^{q}\right\rangle \simeq n^{\nu(q)},
$$

where the exponent $\nu(q)$ has a piecewise linear behavior

$$
\nu(q)=\left\{\begin{array}{ll}
q / 2 \quad \text { if } q<2 \alpha, \\
q-\alpha & \text { if } q>2 \alpha,
\end{array} \quad \alpha=\frac{\gamma+3}{\gamma-1},\right.
$$

in agreement with numerical results shown in Fig. 9. The transition at $q=2 \alpha$ in the momenta spectrum of Eq. (38) is general in systems manifesting anomalous diffusion [25].

As an outcome, we have that (anomalous) transport properties fully agree with the power laws we deduced for the waiting time distribution [Eq. (26)].

\section{NOISE EFFECTS}

In order to better understand the link between correlation decay and time statistics (and to verify, if not rigorously prove, it), we consider the effects of a small stochastic perturbation. The behavior, under the modified dynamics, of the survival probability and of correlation decay may provide further information about the interconnection between them. At the same time, the dynamical effects of a superimposed noise are interesting by themselves (see Refs. [16,37,47,48]).

The general expectation is that small-scale stochasticity blurs the behavior in the vicinity of the parabolic fixed point, enhancing the chaotic character of the motion; one then expects a transition to an exponential decay of the survival probability and correlation functions: this intuition is corroborated by numerical experiments, reported in Fig. 10. We perturb the system by introducing a stochastic noise, adding at each iteration of the map a random vector of the type $\xi$ $=\left(\xi_{1}, \xi_{2}\right)$ with $\xi_{i}$ independent identically distributed (i.i.d) in 


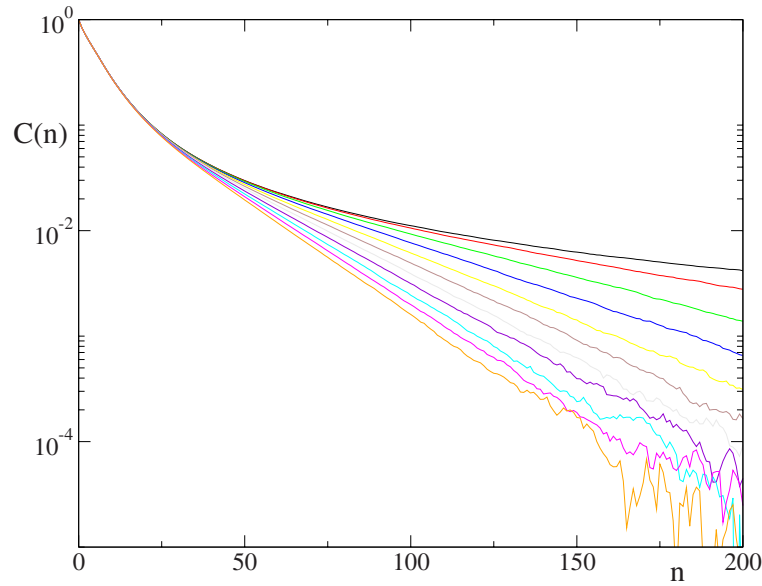

FIG. 10. (Color online) Correlation decay for noisy dynamics, for $\gamma=10$ and various values of $\epsilon$ (from top to bottom: $\epsilon=0.0$, $0.005,0.010,0.015,0.020,0.025,0.030,0.035,0.040,0.045$, and $0.050)$. Each correlation function is computed by considering 3 $\times 10^{9}$ initial conditions.

$(-\epsilon, \epsilon)$. The effects of the perturbation are expected to be dominant in the region of phase space (which will depend on the noise intensity $\epsilon$ and on the stickiness parameter $\gamma$ ) where the deterministic step is small compared to noise. Based on this assumption, we are able to give an analytical estimate of the crossover time $t_{c}$ (defined as the characteristic time of the asymptotic exponential decay), which is in good agreement with numerical simulations.

Following [47], we divide the phase space into two complementary sections: one small region surrounding the fixed point, where the dynamics is dominated by stochastic diffusion, and its complement, far from the fixed point, where the dynamics is dominated by the deterministic chaotic motion.

Now the main problem is a proper definition of the boundary of such a partition. The criterion suggested in [47] consists in defining $\left\langle T_{\text {deterministic }}\right\rangle$ as the mean exit time from the region, say $\Delta$, evaluated with the assumption that the dynamics is only due to the deterministic motion of the unperturbed map; then we define $\left\langle t_{\text {random }}\right\rangle$ as the mean exit time evaluated as if the dynamics were only due to diffusion.

The border of the region is determined by the constraint

$$
\left\langle T_{\text {deterministic }}\right\rangle \simeq\left\langle t_{\text {random }}\right\rangle .
$$

We restrict the analysis to the first quadrant and choose as region $\Delta$ the area defined by the survival probability $p_{\Omega}(k)$, for some time $k$. In this way $\left\langle T_{\text {deterministic }}^{k}\right\rangle$ is given by

$$
\left\langle T_{\text {deterministic }}^{k}\right\rangle=\frac{1}{p_{\Omega}(k)} \sum_{n \geq k}^{\infty} \mu\left(\Omega_{n}\right)(n-k+1) .
$$

Performing the calculation by substituting the probabilities obtained in the previous sections [Eqs. (7) and (8)], we get

$$
\left\langle T_{\text {deterministic }}^{k}\right\rangle \simeq k \text {. }
$$

The calculation of $\left\langle t_{\text {random }}\right\rangle$ is performed as follows. First we approximate $p_{\Omega}(k)$ as in Sec. III with the rectangular regions of Eq. (24), say $x_{k} y_{k}$; this rectangular region can be exited

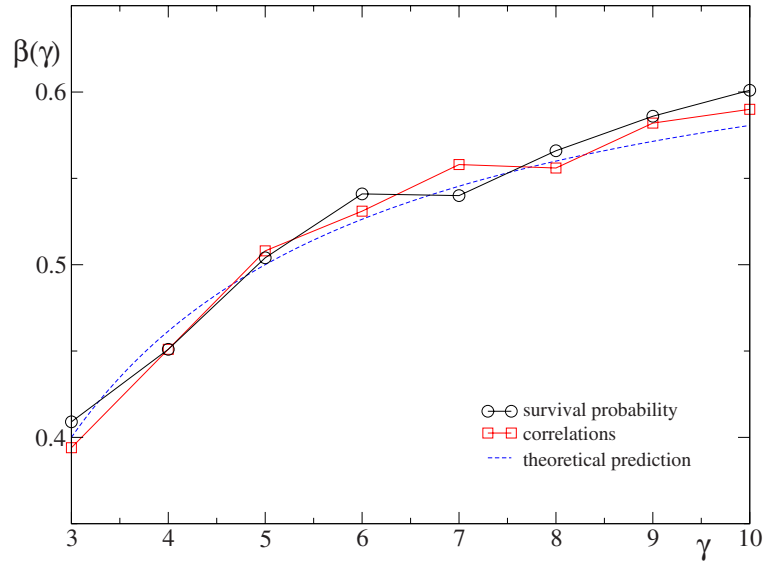

FIG. 11. (Color online) Comparison between theory and numerical results for the function $\beta(\gamma)$ of Eq. (47). Circles, numerical data for the survival probability; squares, numerical data for the correlations; dashed line, theoretical prediction.

along the $x$ or $y$ direction, independently (thanks to the particular form of our noise), so that

$$
\left\langle t_{\text {random }}^{k}\right\rangle=\min \left(\left\langle t_{\text {random }}^{k, x}\right\rangle,\left\langle t_{\text {random }}^{k, y}\right\rangle\right) \text {. }
$$

From the diffusion equation describing stochastic dynamics (see $[47,49]$ ), we get

$$
\left\langle t_{\text {random }}^{k, z}\right\rangle \simeq \frac{z_{k}^{2}}{\epsilon^{2}}
$$

where $z$ can be either $x$ or $y$. Remembering that close to the origin the motion follows dynamics on the unstable manifold and from Eqs. (23) and (24) we get

$$
\begin{gathered}
x_{k} \sim \ell_{k} \sim k^{-2 /(\gamma-1)}, \\
y_{k} \sim x_{k}^{\sigma} \sim k^{-(\gamma+1) /(\gamma-1)} .
\end{gathered}
$$

By substituting Eq. (44) in Eq. (43), we obtain

$$
\left\langle t_{\text {random }}^{k}\right\rangle \simeq \min \left(\frac{\left(k^{-2 /(\gamma-1)}\right)^{2}}{\epsilon^{2}}, \frac{\left(k^{-(\gamma+1) /(\gamma-1)}\right)^{2}}{\epsilon^{2}}\right) .
$$

Finally, from Eq. (39),

$$
k \sim \frac{\left(k^{-(\gamma+1) /(\gamma-1)}\right)^{2}}{\epsilon^{2}} .
$$

Writing the characteristic time of the exponential decay as a function of the noise strength $\epsilon$ and the intermittency parameter $\gamma$, we derive an estimate for the crossover time $t_{c}$ :

$$
t_{c}=k \sim \epsilon^{-\beta(\gamma)} \sim \epsilon^{-(2 \gamma-2) /(3 \gamma+1)} .
$$

Eventually we compare the behavior of $\beta(\gamma)$ with the numerical results, for the survival probability and for the correlation decay.

Numerical results are obtained in the following way. For each value of $\gamma$ we consider either the survival probability or the correlation functions for several values of $\epsilon$ (see Fig. 10): the exponential decay rates, which we consistently observe, are then fitted according to a power law in $\epsilon$. The nice re- 
semblance between the numerical simulations for the correlation decay and the survival probability, together with a good agreement with the analytical result of Eq. (47) (see Fig. 11). strengthen our belief that the two distributions in the unperturbed case should be driven by the same exponent.

\section{CONCLUSIONS}

We considered a one-parameter family of area-preserving maps which generalizes intermittent behavior in two dimensions (while admitting a smooth invariant measure). This is a paradigmatic example of weak chaos for Hamiltonian maps even if the measure of the "regular" portion of the phase space is zero (only a parabolic fixed point): the parameter $\gamma$ controls sticking of trajectories to the fixed point. By considering the motion along the unstable manifold close to the fixed point, we are led to estimates for power-law decays of the survival probability and waiting time distribution. Numerical computations of survival probabilities and residence time statistics show a very close agreement with predicted exponents. Correlation functions are harder to deal with numerically, yet in this case the results are close to analytical predictions. Our results are further supported by considering transport properties for a lift of the map, and by studying dynamical effects induced by a stochastic perturbation.

While we think that a complete quantitative understanding of weak chaos in more than one dimension is still in its infancy, our results fortify the connection of local features (motion along the unstable manifold close to the fixed point) to global dynamical quantities, such as mixing speed, transport properties, and response to stochastic perturbations.

\section{ACKNOWLEDGMENTS}

This work has been partially supported by MIUR-PRIN projects "Transport Properties of Classical and Quantum Systems" and "Quantum Computation with Trapped Particle Arrays, Neutral and Charged." We thank Raffaella Frigerio and Italo Guarneri for sharing their early work on the problem, and Carlangelo Liverani and Sandro Vaienti for useful discussions and information.
[1] L. Markus and K. R. Meyer, Mem. Am. Math. Soc. 144, 1 (1974).

[2] J. D. Meiss, Rev. Mod. Phys. 64, 795 (1992).

[3] T. Geisel and S. Thomae, Phys. Rev. Lett. 52, 1936 (1984).

[4] C. F. F. Karney, Physica D 8, 360 (1983).

[5] B. V. Chirikov and D. L. Shepelyansky, Physica D 13, 395 (1984).

[6] F. Vivaldi, G. Casati, and I. Guarneri, Phys. Rev. Lett. 51, 727 (1983).

[7] G. M. Zaslavsky and B. A. Niyazov, Phys. Rep. 283, 73 (1997).

[8] R. Artuso and G. Cristadoro, in Anomalous Transport: Foundations and Applications, edited by R. Klages, G. Radons, and I. M. Sokolov (Wiley-VCH, Weinheim, 2007).

[9] A. J. Lichtenberg and M. A. Lieberman, Regular and Chaotic Dynamics (Springer-Verlag, New York, 1992).

[10] R. Artuso and R. Prampolini, Phys. Lett. A 246, 407 (1998).

[11] R. Frigerio, Laurea thesis, Università dell'Insubria, 2000; R. Frigerio and I. Guarneri (unpublished).

[12] C. Liverani and M. Martens, Commun. Math. Phys. 260, 527 (2005)

[13] Y. Pomeau and P. Manneville, Commun. Math. Phys. 74, 189 (1980).

[14] P. Gaspard and X.-J. Wang, Proc. Natl. Acad. Sci. U.S.A. 85, 4591 (1988); X.-J. Wang, Phys. Rev. A 40, 6647 (1989).

[15] L.-S. Young, Isr. J. Math. 110, 153 (1999).

[16] C. Liverani, B. Saussol, and S. Vaienti, Ergod. Theory Dyn. Syst. 19, 671 (1999).

[17] R. Zweimüller, Stochastics Dyn. 3, 83 (2003).

[18] H. Hu and S. Vaienti (unpublished).

[19] R. Artuso, P. Cvitanović, and G. Tanner, Prog. Theor. Phys. Suppl. 150, 1 (2003); R. Artuso, P. Dahlqvist, G. Tanner, and P. Cvitanović, in Classical and Quantum Chaos, edited by P. Cvitanović, R. Artuso, R. Mainieri, G. Tanner, and G. Vattay
(Niels Bohr Institute Press, Copenhagen, 2005).

[20] S. Isola, Nonlinearity 15, 1521 (2002); T. Prellberg, J. Phys. A 36, 2455 (2003); S. Tasaki and P. Gaspard, J. Stat. Phys. 109, 803 (2002).

[21] T. Miyaguchi and Y. Aizawa, Phys. Rev. E 75, 066201 (2007).

[22] S. Gouëzel, Isr. J. Math. 139, 29 (2004).

[23] T. Geisel, J. Nierwetberg, and A. Zacherl, Phys. Rev. Lett. 54, 616 (1985).

[24] A. S. Pikovsky, Phys. Rev. A 43, 3146 (1991).

[25] P. Castiglione, A. Mazzino, P. Muratore-Ginanneschi, and A. Vulpiani, Physica D 134, 75 (1999).

[26] R. Artuso and G. Cristadoro, Phys. Rev. Lett. 90, 244101 (2003).

[27] M. Pollicott and M. Yuri, Commun. Math. Phys. 217, 503 (2001).

[28] We point out that it is possible to define smooth functions $f$ reproducing each integer $\gamma$ behavior; this was accomplished in [11].

[29] J. D. Meiss, Chaos 7, 139 (1997).

[30] S. R. Channon and J. L. Lebowitz, Ann. N.Y. Acad. Sci. 357, 108 (1980).

[31] P. Dahlqvist and R. Artuso, Phys. Lett. A 219, 212 (1996).

[32] R. Artuso, G. Casati, and I. Guarneri, J. Stat. Phys. 83, 145 (1996).

[33] S. Isola, in Nonlinear Dynamics of Electronic Systems, edited by G. Setti, R. Rovatti, and G. Mazzini (World Scientific, Singapore, 2000).

[34] G. Casati and T. Prosen, Phys. Rev. Lett. 85, 4261 (2000).

[35] R. Artuso and G. Cristadoro, J. Phys. A 37, 85 (2004).

[36] I. Procaccia and H. Schuster, Phys. Rev. A 28, 1210 (1983).

[37] J. E. Hirsch, B. A. Huberman, and D. J. Scalapino, Phys. Rev. A 25, 519 (1982).

[38] P. Dahlqvist, Phys. Rev. E 60, 6639 (1999).

[39] J. D. Crawford and J. R. Cary, Physica D 6, 223 (1983). 
[40] P. Collet and S. Isola, Commun. Math. Phys. 139, 551 (1991).

[41] H. Scher and E. W. Montroll, Phys. Rev. B 12, 2455 (1975).

[42] G. Zumofen and J. Klafter, Phys. Rev. E 47, 851 (1993).

[43] K. H. Andersen, P. Castiglione, A. Mazzino, and A. Vulpiani, Eur. Phys. J. B 18, 447 (2000).

[44] I. M. Sokolov, Phys. Rev. E 63, 011104 (2000).

[45] J. Klafter, A. Blumen, and M. F. Shlesinger, Phys. Rev. A 35, 3081 (1987).
[46] W. Feller, An Introduction to Probability Theory and Applications (Wiley, New York, 1966), Vol. II.

[47] E. Floriani, R. Mannella, and P. Grigolini, Phys. Rev. E 52, 5910 (1995).

[48] E. G. Altmann and H. Kantz, Europhys. Lett. 78, 10008 (2007).

[49] N. Agmon and G. H. Weiss, J. Phys. Chem. 93, 6884 (1989). 